\title{
Role of Helicobacter pylori surface structures in bacterial interaction with macrophages
}

\author{
M Chmiela, E Czkwianianc, T Wadstrom, W Rudnicka
}

\begin{abstract}
Background-Helicobacter pylori infection is associated with a marked infiltration of the gastric epithelium by neutrophils, macrophages, lymphocytes, and plasma cells. Despite the presence of phagocytes in close vicinty to $H$ pylori microbes a great number of people are unable to eradicate bacteria.

Aims-To investigate the involvement of multiple bacterial 'adhesins' and some phagocytic receptors in the process of the ingestion of $\mathrm{H}$ pylori by macrophages.

Bacteria-H pylori strains differing in the expression of sialic acid dependent (sHA) or sialic acid independent (HA) haemagglutinin and heparan sulphate binding were selected for the study.
\end{abstract}

Methods-The uptake of fluorescein labelled $H$ pylori bacteria by a homogenous macrophage cell line $\mathrm{J}$ 774A.1 was estimated in a quantitative fluorometric assay.

Results-The ingestion of $H$ pylori 17874 and 25 strains expressing sHA was inhibited by the pretreatment of the bacteria with anti-sHA antibodies or fetuin as well as by treatment of the macrophages with neuraminidase. In contrast the uptake of $H$ pylori 17875 strain expressing HA remained unchanged. The phagocytosis of all investigated bacteria was inhibited after the treatment with heparin, hyaluronic acid or vitronectin with fresh but not heat inactivated serum.

Conclusions-The results suggest that $H$ pylori surface compounds binding host proteins such as fetuin, heparin/haparan sulphate, hyaluronic acid, and vitronectin in the presence of complement, could allow the bacteria to avoid phagocytosis.

(Gut 1997; 40: 20-24)

Keywords: Helicobacter pylori, macrophages, fetuin, heparin, vitronectin.

Helicobacter pylori has been recognised as a common case of chronic, active type $B$ gastritis and peptic ulcer disease. The bacteria were also considered to constitute a risk factor for the development of gastric carcinoma and gastric lymphoma. ${ }^{1}$ As a result of $H$ pylori induced inflammatory response an accumulation of neutrophils in gastric mucosa is followed by a marked infiltration with macrophages as well as lymphocytes and plasma cells. ${ }^{23}$ Despite mobilisation of numerous phagocytes to inflammatory foci in gastric mucosa a great number of people are not able to eliminate the bacteria. In this context, it is interesting to consider how $H$ pylori bacteria evade destruction by phagocytic cells. It has been established that $H$ pylori strains express surface haemagglutinins, ${ }^{4}$ and heparan sulphate binding proteins, ${ }^{6} 7$ and that they differ in the expression of these constituents. Recently, we showed that these structures are involved in the interaction of $H$ pylori bacteria with phagocytes. ${ }^{8}$ Human as well as murine neutrophils and macrophages seemed to recognise mainly haemagglutinins on the surface of some $H$ pylori strains and heparan sulphate binding proteins on other strains of this species. ${ }^{9}$ Frequently the attachment of the microorganisms to phagocytes leads to stimulation, ingestion, and killing. However, we observed very strong attachment to the macrophages and a comprehensive resistance to intracellular killing of a $\mathrm{H}$ pylori strain 17874 expressing sialic acid dependent haemagglutinin. ${ }^{10}$ The aim of this study was to investigate in detail the involvement of multiple 'adhesins' of $H$ pylori bacteria and some phagocytic receptors in the process of the ingestion of $H$ pylori strains by macrophages.

\section{Methods}

Bacterial strains

$H$ pylori strains 17874 (NCTC 11637) and 17875 (NCTC 11638) were from the Culture Collection University of Gothenburg, Sweden, and $H$ pylori 25 strain was a clinical isolate from adenocarcinoma vertical case. The $H$ pylori strains 17874 and 25 produce sialic acid dependent and $H$ pylori strain 17875 sialic acid independent haemagglutinins. ${ }^{4}$ Although all bacterial strains used bind heparan sulphate, the $H$ pylori 25 demonstrates a comparatively strong and the $H$ pylori 17874 comparatively weak heparan sulphate binding activity. ${ }^{6}$ The bacteria were grown on $5 \%$ horse blood agar for $36-48$ hours at $37^{\circ} \mathrm{C}$ under microaerophilic conditions.

\section{Labelling of bacteria}

The bacteria were heat killed $\left(60^{\circ} \mathrm{C}\right.$ for 30 minutes), washed once with phosphate buffered saline (PBS) (150 mM NaCl, 3.4 mM $\mathrm{KCl}, 10.1 \mathrm{mM} \mathrm{Na} \mathrm{HPO}_{4}, 1.8 \mathrm{mM} \mathrm{KH}_{2} \mathrm{PO}_{4}$; pH 7.3), and resuspended in carbonate buffered saline (CBS) (70 mM Na $\mathrm{CO}_{3}, 93 \mathrm{mM}$ $\mathrm{NaHCO}_{3}, 154 \mathrm{mM} \mathrm{NaCl}: \mathrm{pH} 9 \cdot 0$ ) at $1 \times 10^{9}$ cells $/ \mathrm{ml}$. To a bacterial suspension (on ice) an equal volume of $1 \%$ DMSO in CBS containing $100 \mu \mathrm{g} / \mathrm{ml}$ fluorescein isothiocyanate (FITC; Sigma St Louis, US) was added. The suspension was agitated for 30 minutes at room 
temperature. The bacteria, after washing with PBS, were resuspended in CBS containing 4\% bovine serum albumin (CBS-BSA) to bind unconjugated FITC to BSA. After 15 minutes at room temperature, the bacteria were washed with CBS-BSA, PBS, and finally with RPMI1640 medium. Labelled bacteria were suspended in RPMI- 1640 medium with $20 \%$ fetal calf serum (FCS) at $1 \times 10^{9}$ cells $/ \mathrm{ml}$. They could be stored at $4^{\circ} \mathrm{C}$ for two weeks without loss of fluorescence. Before use in the phagocytosis assay, FITC labelled bacteria were centrifuged and resuspended at $1 \times 10^{8}$ cells $/ \mathrm{ml}$ in RPMI-1640 medium.

\section{Modulation of bacterial surface}

FITC labelled bacteria in RPMI-1640 medium $\left(200 \mu \mathrm{l}, 2 \times 10^{9} / \mathrm{ml}\right)$ were mixed with $200 \mu \mathrm{l}$ of: fetuin, asialofetuin, heparin, hyaluronic acid $(1000 \mu \mathrm{g} / \mathrm{ml}$ in RPMI-1640 medium; Sigma), vitronectin $(100 \mu \mathrm{g} / \mathrm{ml}$ in RPMI-1640 medium; Polyclone, Poland) alone or vitronectin with fresh or heat-inactivated $\left(56^{\circ} \mathrm{C}, 30 \mathrm{~min}\right)$ normal rabbit serum or rabbit antiserum against sHA $(1: 10)$. Bacterial suspensions were agitated for one hour at $37^{\circ} \mathrm{C}$ and then distributed $\left(100 \mu \mathrm{l}\right.$ containing $1 \times 10^{7}$ bacterial cells) into the wells of a microplate containing macrphages.

\section{Macrophages}

The macrophage cell line J774A.1 (ATCC, Rockville, MD) was used. The cells were grown in RPMI-1640 medium supplemented with $10 \%$ heat inactivated FCS, $2 \mathrm{mM}_{\mathrm{L} \text {-glutamine }}$ and $50 \mu \mathrm{g} / \mathrm{ml}$ gentamicin, for three to four days at $37^{\circ} \mathrm{C}$ in a humidified $5 \% \mathrm{CO}_{2}$ atmosphere. For phagocytosis assay, $1 \times 10^{5}$ macrophages in $100 \mu \mathrm{l}$ RPMI-1640 medium containing 10\% FCS was placed in each well of flat bottom 96 well microplates (Falcon, Becton, Dickinson, $\mathrm{CA}$ ) and allowed to adhere to the plastic within 18 hours $\left(37^{\circ} \mathrm{C}, 5 \% \mathrm{CO}_{2}\right)$. Before the assay the monolayers were washed three times with RPMI-1640 medium.

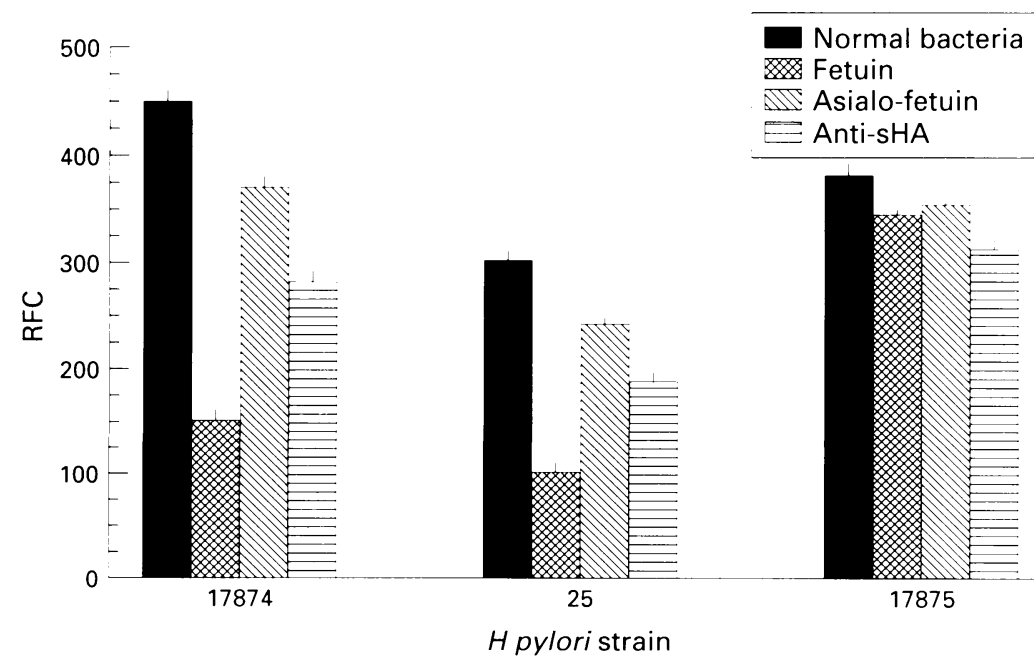

Figure 1: Sialic acid residue dependent phagocvtosis of $\mathrm{H}$ pylori. The uptake of FITC labelled bacteria, normal or coated with fetuin, asialofetuin or rabbit anti-sHA antibodies was estimated by a quantitative fuorometric assay. The number of ingested bacteria was expressed as relative fluorescence units $(R F U)$, mean (SD).
In some wells, the macrophages were preincubated for 30 minutes $\left(37^{\circ} \mathrm{C}, 5 \% \mathrm{CO}_{2}\right)$ with neuraminidase $(0.3 \mathrm{U} / \mathrm{ml})$ to remove surface sialic acid components or with $\mathrm{Fc}$ fragment of mouse IgG $(10 \mu \mathrm{g} / \mathrm{ml})$ to block macrophage Fc receptors. The viability of pretreated and untreated macrophages was in the range of $95-97 \%$ as estimated by trypan blue exclusion. Determination of the number of macrophages bound to the wells at the beginning and the end of phagocytosis assay was based on selective staining of the phagocyte nuclei with methylene blue (Sigma), followed by extraction of the stain. ${ }^{11}$ In each experiment the macrophages pre-incubated for 60 minutes $\left(37^{\circ} \mathrm{C}, 5 \% \mathrm{CO}_{2}\right)$ with cytochalasin $\mathrm{D}(1.0 \mathrm{mg} / \mathrm{ml}$; dissolved in DMSO and diluted with RPMI-1640 medium) were used in parallel with untreated cells. Pretreatment of macrophages with cytochalasin D completely inhibited phagocytic activity of the cells without affecting their viability.

\section{Phagocytosis assay 1213}

The $100 \mu \mathrm{l}$ aliquots of the FITC labelled bacterial suspension $\left(1 \times 10^{8}\right.$ cells $\left./ \mathrm{ml}\right)$ were added to the macrophage monolayers. The cell cultures were incubated for one hour at $37^{\circ} \mathrm{C}$, $5 \% \mathrm{CO}_{2}$, and the medium was removed from the wells. Extracellular fluorescence was quenched with $100 \mu \mathrm{l} /$ well of $0 \cdot 2 \%$ trypan blue in PBS. The wells containing FITC labelled bacteria alone were used as control of quenching effectiveness. The intensity of fluorescence was determined in relative fluorescence units (RFU), at $485 \mathrm{~nm}$ excitation and $530 \mathrm{~nm}$ emission wavelengths, in a fluorescence reader (Microplate fluorometer Model 7620, Cambridge Technology, Watertown, MA) interfaced with a PC compatible computer.

In each experiment standard curves for quantitating FITC labelled $H$ pylori strains were prepared. $H$ pylori bacteria suspensions were serially diluted in RPMI-1640 medium, distributed into microplate wells, and the fluorescence of the bacteria was measured. The values for fluorescence were plotted as a function of the number of bacteria in each well.

Statistical analysis

Data are expressed as (SD). Statistical significance was determined by Student's two tailed $t$ test and defined as $\mathrm{p}$ value.

\section{Results}

Sialic acid residues dependent ingestion of $\mathrm{H}$ pylori by macrophages

The involvement of the surface sialylated structures in the ingestion of $H$ pylori bacteria was investigated in two ways: by blocking bacterial ligands recognised by macrophage receptors containing sialic acid residues and by removing such receptors from the macrophage surface. Figure 1 shows a dramatic decrease (near 70\%) 


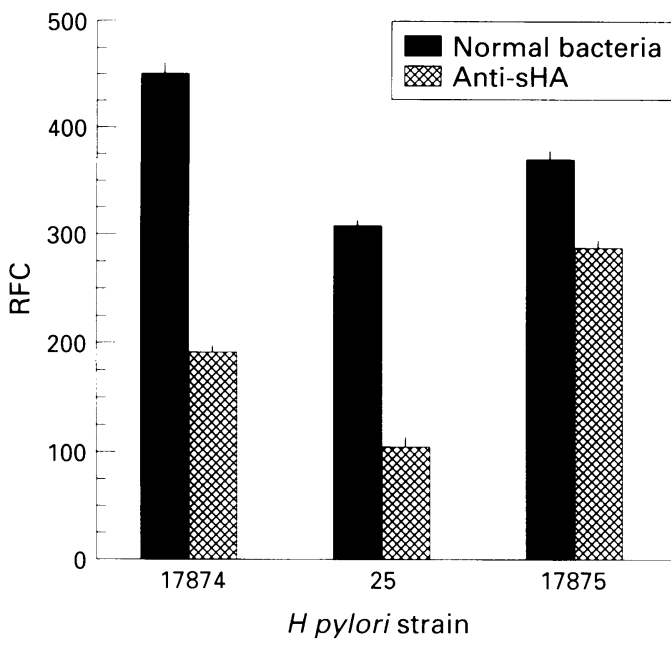

Figure 2: Sialic acid residue dependent phagocytosis of $\mathrm{H}$ pylori. The uptake of FITC labelled bacteria (normal or coated with anti-sHA antibodies):by normal or Fc IgGtreated macrophages was estimated by fluorometric assay.

The number of ingested organisms was expressed as relative fluorescence units (RFU), mean (SD).

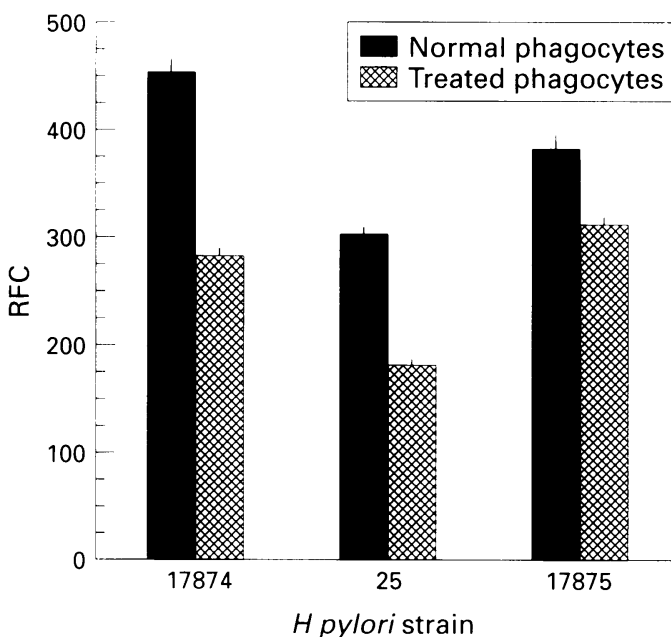

Figure 3: Sialic acid residue dependent phagocytosis of $\mathrm{H}$ pylori. The ingestion of FITC labelled bacteria by normal or neuraminidase treated macrophages was estimated by fluorometric assay. The number of ingested microbes was expressed as relative fluorescence units (RFU), mean (SD).

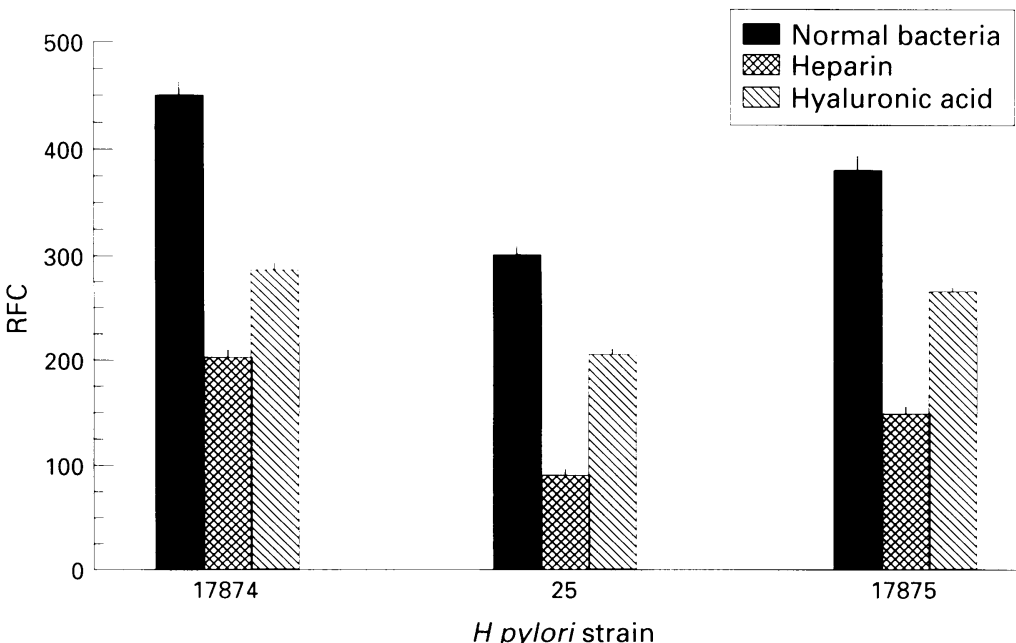

Figure 4: The role of glycosaminoglycans in the ingestion of $\mathrm{H}$ pylori by macrophages. The uptake of FITC labelled bacteria, normal or coated with heparin or hyaluronic acid was estimated by fluorometric assay. The number of ingested organisms was expressed as relative fluorescence units (RFU), mean (SD). in the ingestion of fetuin treated $H$ pylori 17874 and 25 strains compared with untreated bacteria. The preincubation of those strains with asialofetuin only slightly reduced (about $25 \%$ ) their ingestion by the macrophages. Both $H$ pylori 17874 and 25 strains express sHA and anti-sHA antibody diminished the engulfment of those bacteria by normal macrophages by about $40 \%$ (Fig 1). However, the inhibitory effect of preincubation of $H$ pylori 17874 and 25 strains with anti-sHA antibody was increased to about $60 \%$ when the macrophages were treated with the Fc fragments of mouse IgG before use in the phagocytosis assay (Fig 2). In contrast with $H$ pylori 17874 and $25, H$ pylori 17875 bacteria expressing a sialic acid independent haemagglutinin were ingested by normal macrophages in the same numbers before and after the treatment with fetuin or anti-sHA antibody (Fig 1). Although, Fc fragments treated macrophages engulfed slightly less $(\mathrm{p}=0.0984)$ anti-sHA treated $H$ pylori 17875 microorganisms than untreated bacteria (Fig 2). The difference was marginally significant.

The removing of sialic acid residues from the macrophage surface by neuraminidase decreased their ability to ingest $H$ pylori 17874 and 25 bacteria by about $40 \%$ and $H$ pylori 17875 microbes by $20 \%$ (Fig 3 ).

Role of glycosaminoglycans in ingestion of $\mathrm{H}$ pylori by macrophages

Figure 4 shows the decrease in the susceptibility to the ingestion by macrophages of all three $H$ pylori strains treated with heparin. This decrease was slightly less evident in the case of $H$ pylori 17874 (inhibition 55\%) expressing comparatively weak heparan sulphate binding activity than in the case of $H$ pylori 25 (inhibition $70 \%$ ) showing relatively high heparan sulphate adhesion. The engulfment of hyaluronic acid treated $H$ pylori bacteria by macrophages was diminished by about $30 \%$.

Influence of vitronectin and complement on phagocytosis of $\mathrm{H}$ pylori

Data for $H$ pylori 17874 strain were chosen for presentation (Fig 5). The results for three different $H$ pylori strains were similar. The preincubation of $H$ pylori microorganisms with vitronectin alone slightly reduced $(p=0.0270)$ and the pretreatment of the bacteria with fresh serum slightly increased $(p=0.0008)$ the phagocytosis (Fig 5). However, the preincubation of the bacteria in the medium containing both vitronectin and fresh serum decreased their ingestion by $46 \%-67 \%$. No inhibition of the phagocytosis was observed for the bacteria preincubated in the medium containing vitronectin and heat inactivated serum.

\section{Discussion}

Phagocytic cells form an important defence against invading microorganisms. These cells recognise and subsequently ingest and kill several infectious agents. Moreover, numerous 


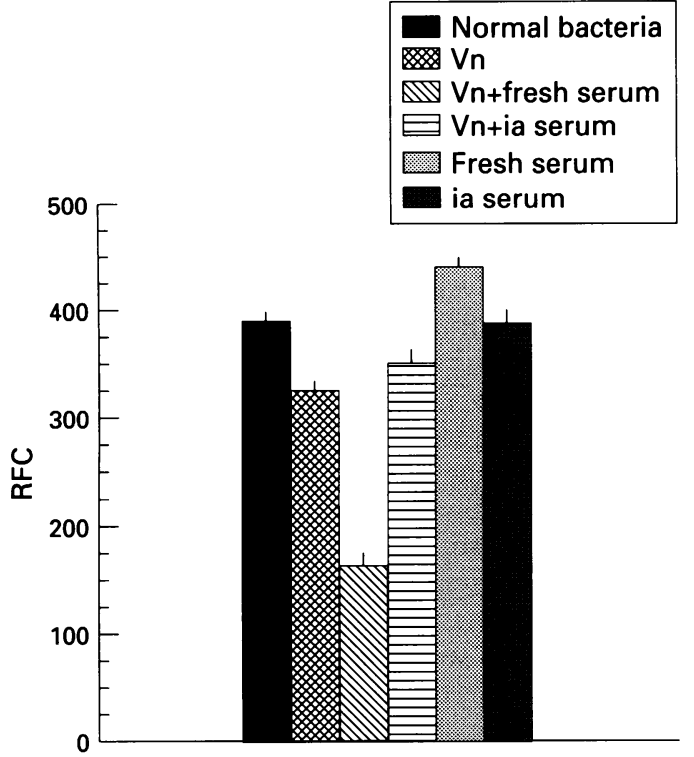

H pylori 17874

Figure 5: The influence of vitronectin and complement on phagocytosis of $\mathrm{H}$ pylori. The FITC labelled bacteria, normal or preincubated with vitronectin $(V n)$ were incubated with macrophages in medium containing both vitronectin and fresh rabbit serum (Vn+fresh serum) or fresh serum alone (fresh serum), vitronectin and heat inactivated serum (Vn+ia serum) or inactivated serum alone (ia serum). The number of ingested bacteria was estimated fluorometrically and expressed as relative fluorescence units (RFU), mean (SD).

cytokines released by bacteria activated phagocytes regulate the effectiveness of the immune network. In the process of phagocytosis, a recognition of microorganisms by neutrophils and macrophages plays a crucial part. In general, two molecular mechanisms of recognition of microbes by phagocytes are distinguished: opsonin dependent (indirect) and opsonin independent (direct). ${ }^{14}$ The opsonins such as antimicrobial IgG or the $\mathrm{C} 3 \mathrm{bi}$ fragments of $\mathrm{C} 3$ component of complement form the bridges by binding to the surface of the microorganisms at one end and to specific receptors, $\mathrm{FcR}$ or $\mathrm{C} 3 \mathrm{R}$ respectively, on the phagocyte surface. The importance of opsonic activity of the complement in the ingestion of $H$ pylori bacteria by neutrophils was shown by McKinlay et al. ${ }^{15}$ On the other hand, Rautelin et al showed that about one third of $H$ pylori strains isolated from human gastric biopsy specimens induced strong chemiluminescence in neutrophils even without serum opsonins. ${ }^{16}$ For other strains complement was required, although even then an oxidative burst induced by them remained at a low level. The bacteriaphagocyte interaction is also regulated by serum opsonins, named collectins, such as surphactant proteins, $C$ reactive protein, and mannose binding protein. These proteins combine with complementary carbohydrates on the microorganisms and with special collectin receptors in the phagocytes. ${ }^{17}$ In contrast with opsonic phagocytosis, the molecular mechanisms of opsonin independent phagocytosis remain obscure. However, three forms of direct recognition of the microorganisms by the phagocytes have been described: lectinophagocytosis based on recognition between surface lectins on one cell (microbial or phagocytic) and surface carbohydrates on the interacting cell, protein-protein interactions between the Arg-Gly-Asp (RGD) sequence of microorganisms and phagocytic integrins and hydrophobic interactions between microbes and phagocytes. ${ }^{14}$

In this study, the molecular basis of nonopsonic phagocytosis of $H$ pylori microorganisms by the macrophages was rather complex as judged by the pattern of inhibition. By using a homogenous macrophage cell line we confirmed the differentiation of $H$ pylori strains with regard to the surface structures regulating their interactions with the macrophages. Covering of surface sialic acid haemagglutinin on H pylori 17874 (NCTC 11637) and 25 strains by treatment with fetuin or specific anti-sHA antibodies inhibited the ingestion of those bacteria by macrophages. The binding of gold labelled fetuin to the surface of some $H$ pylori strains was shown by Kobayashi et al as well as the inhibitory effect of fetuin on the attachment of such strains to Hep-2 cells derived from human larynx carcinoma. ${ }^{18}$ As we showed in this study, the inhibitory effect of fetuin on the ingestion of $H$ pylori strains expressing sHA was stronger compared with the inhibition caused by anti-sHA antibodies. This difference could be explained by an increase in phagocytosis of $H$ pylori by $\mathrm{Fc}$ fragments of anti-sHA antibodies that are recognised by $\mathrm{FcR}$ receptors of the macrophages. These results also suggested cooperative action of both sHA-mediated nonopsonic phagocytosis and FcR mediated opsonic phagocytosis of $H$ pylori bacteria. The inhibition of the engulfment of $H$ pylori 17874 and 25 bacteria by macrophage treated with neuraminidase additionally proved the role of the interaction between bacterial sHA and macrophage sialic acid residues in the recognition of sHA positive $H$ pylori strains. However, the process of recognition of $H$ pylori by the macrophages was more complex and it invovled the interaction between glycosaminoglycans expressed on the surface of the macrophages and surface bacterial protein binding heparin. The saturation of such proteins by the treatment of $H$ pylori bacteria with heparin caused a dramatic decrease in the phagocytosis. A similar phenomenum was described for Leishmania amastigotes. ${ }^{19}$ The ingestion of $H$ pylori strains was also inhibited by covering the bacteria with hyaluronic acid. This hydrophilic glycosaminoglycan could inhibit the uptake of $H$ pylori bacteria by decreasing their surface hydrophobicity. It is known that more hydrophobic bacteria are ingested to the largest extent. ${ }^{20}$

The process of $H$ pylori ingestion was also influenced by vitronectin and complement. Although vitronectin alone slightly reduced and fresh serum as a source of complement slightly increased the uptake of the bacteria by macrophages, the simultaneous treatment of the bacteria with vitronectin and complement made them quite resistant to phagocytosis. Vitronectin (or S- protein) is a highly glycosylated protein of serum, tissues and extracellular matrices, characterised as a cell attachment 
factor. ${ }^{21}$ This glycoprotein binds to many bacteria among them to $H$ pylori microorganisms. ${ }^{22}$ During complement activation, vitronectin is incorporated into $\mathrm{C} 5 \mathrm{~b}-\mathrm{C} 7 \mathrm{com}$ plex preventing formation of the membrane attack complex of complement on a macrophage surface..$^{2123}$ Therefore, vitronectin binding bacteria can avoid bacteriolysis. On the other hand, soluble S-C5b-C7 complexes as well as $\mathrm{S}-\mathrm{C} 5 \mathrm{~b}-\mathrm{C} 9$ complexes can bind to macrophage vitronectin receptors ${ }^{24}$ and heparin or heparan sulphate structures on macrophages. It has been shown that vitronectin as well as C6, C8 and C9 subunits of complement bind to heparin. ${ }^{25}$ If so, such complexes could prevent the interaction between macrophage glycosaminoglycans and bacterial heparin binding proteins. As we have shown in this paper, this interaction was crucial for $H$ pylori phagocytosis and the preincubation of the bacteria with heparin inhibited the ingestion of the bacteria by $55 \%-70 \%$.

In conclusion, this study shows that the ingestion of $H$ pylori bacteria by the macrophages is a cooperative process involving both non-opsonic and opsonic mechanisms. However, direct, non-opsonic phagocytosis seems to be a particularly effective way for the uptake of $H$ pylori bacteria by the macrophages and considerable strain differences in the expression of structures recognised by macrophages are found. Evidently, a deposition of sialic acid containing compounds, heparin or vitronectin in the presence of complement on bacterial surface, made the bacteria resistant to the phagocytosis. It is probable that in vivo, especially in inflammatory milieu, absorbing of such compounds by $H$ pylori bacteria may allow them to avoid phagocytosis. This may be very important for the persistance of $H$ pylori microorganisms on inflammatory gastric mucosa.

This study was supported by the Swedish Board for Technical Development (STU/NUTEK), a grant from Swedish Medical Research Council (16x04723), and a grant from the Polish Research Committee (KBN No 685/P05/95/09). We thank ou colleagues from the Department of Animal Physiology University of Lund for accessing the fluorescence reader.

1 Wadström T. An update of Helicobacter pylori. Current Opinion in Gastroenterology 1995; 11: 69-75.

2 Mooney C, Keenan J, Munster D. Neutrophil activation by Helicobacter pylori. Gut 1991; 32: 853-7.

3 Sobala GM, Crabtree JE, Dixon F. Acute Helicobacter pylor infection: clinical features, local and systemic response, gastric mucosal histology and gastric juce ascorbic acid concentration. Gut 1991; 32: 1415-8.

4 Lelwala-Guruge J, Ljungh A, Wadström T. Haemagglutination patterns of Helicobacter pylori. APMIS 1992; 100: $908-13$.

5 Taylor NS, Hasubski AT, Fox JG, Lee A. Haemagglutination profiles of Helicobacter species that cause gastritis in man and animals. $₹$ Med Microbiol 1992; 37: 299-303.

6 Ascencio F, Fransson LA, Wadström T. Affinity of the gastric pathogen Helicobacter pylori for the $\mathrm{N}$-sulphated glycosaminoglycan heparan sulphate. $\mathcal{F}$ Med Microbiol 1993; 38: 240-4

7 Hirmo S, Utt $M$, Ringer $M$, Wadström $T$. Inhibition of heparan sulphate and other glycosaminoglycans binding to Helicobacter pylori by various polysulphated carbohydrates. FEMS Immunol Med Microbiol 1995; 10: hydrates.

8 Chmiela M, Lelwala-Guruge J, Wadström T. Interaction of cells of Helicobacter pylori with human polymorphonuclear leukocytes: possible role of haema
Immunol Med Microbiol 1994; 9: 41-8.

9 Chmiela M, Paziak-Domañska B, Rudnicka W, Wadström T. The role of heparan sulphate-binding activity of Helicobacter pylori bacteria in their adhesion to murine macrophages. APMIS 1995; 103: 469-74.

10 Chmiela A, Paziak-Domañska B, Wadström T. Attachment, ingestion and intracellular killing of Helicobacter pylori by human peripheral blood mononuclear leukocytes and mouse peritoneal inflammatory macrophages. FEMS Immunol Med Microbiol 1995; 10: 307-16.

11 Bracha R, Mirelman D. Virulence of Entamoeba histolytica trophozoites. Effects of bacteria, microaerophilic controphozoites. Effects of bacteria, microaerophilic con-
ditions and metronidazole. $₹ \operatorname{Exp}$ Med 1984; 160: ditions

12 Wan Ch P, Park Ch S, Lars BHSA. A rapid and simple microfluorometric phagocytosis assay. $\mathcal{F}$ Immunol Meth 1993; 162: 1-7.

13 Oben JA, Foreman JCA. A simple quantitative fluorometric assay of in vitro phagocytosis in human neutrophils. $\mathcal{F}$ Immunol Meth 1988; 112: 99-103.

14 Ofek I, Goldhar J, Keisari Y. Nonopsonic phagocytosis of microorganisms. Annu Rev Microbiol 1995; 49: 239-76.

15 McKinley AW, Young A, Russell RI, Gemmell CG. Opsonic requirements of Helicobacter pylori. $\mathcal{f}$ Med Microbiol 1993; 38: 209-15.

16 Rautelin H, Blomberg B, Fredlund H, Järnerot G, Danielsson D. Incidence of Helicobacter pylori strains Danielsson D. Incidence of Helicobacter pylori strains
activating neutrophils in patients with peptic ulcer activating neutrophils in patie
disease. Gut 1993; 34: 599-603.

17 Holmskov U, Malhotra R, Sim RB, Jensenius JC. Collectins: collagenous C-type lectins of the innate immune defense system. Immunol Today 1994; 15: 67-74.

18 Kobayashi Y, Okazaki K-I, Murakami K. Adhesion of Helicobacter pylori to gastric epithelial cells in primary cultures obtained from stomachs of various animals. Infect Immun 1993; 61: 4058-63.

19 Love DC, Esko J, Mosser DM. A heparin-binding activity of Leishmania amastigotes which mediates adhesion to of Leishmania amastigotes which mediates adhesion

20 Absolom DR. The role of bacterial hydrophobicity in infection: bacterial adhesion and phagocytic ingestion. infection: bacterial adhesion and

21 Tomasini BR, Mosher DF. Vitronectin. Prog Hemost Thromb 1990; 10: 269-305.

22 Ringer $M$, Pallson $M$, Wadström. Vitronectin binding by Helicobacter pylori. FEMS Immunol Med Microbiol 1992; 7: 29-38.

23 Dohlbäck B, Podach ER. Characterization of human S protein, an inhibitor of the membrane attack complex of complement. Demonstration of a free reactive thiol group. Biochemistry 1985; 24: 2368-74.

24 Savill J, Dransfield I, Hogg N, Naslett CH. Vitronectinreceptor-mediated phagocytosis of cells undergoing receptor-mediated phagocytosis of
apoptosis. Nature 1990; 343: 170-3.

25 Sahu A, Pangburn MK. Identification of multiple sites of interaction between heparin and the complement system. Immunology 1993; 30: 679-84. 